\title{
Recent patent applications in computational biotechnologies
}

\author{
Julien Muzard
}

With the prevalence of genomic and proteomic data, modern research in experimental biotech has become a human-guided, computer-assisted endeavor. A wide range of molecular and structural bioinformatics methods and resources is available and the number of therapeutically relevant macromolecular entities is growing rapidly. This highlights the need for using computer-aided techniques for the identification and efficient optimization of novel hit compounds. Table $\mathbf{1}$ is an attempt to catalog recent patents for in silico prediction tools, compound collections, some ligand-based prediction methods, characterization/simulation of three-dimensional targets and homology modeling tools together with all-atom molecular dynamics methodology for the analysis of macromolecular struc- tures and interfaces. Different applications and approaches facilitating the drug discovery process are reported. This review should help readers find patented computer tools useful for facilitating rapid and cost-effective identification of new hit compounds.

\section{COMPETING FINANCIAL INTERESTS}

The author declares no competing financial interests.

\begin{tabular}{|c|c|c|c|c|c|}
\hline Patent number & Description & Assignee & Inventor & $\begin{array}{l}\text { Priority } \\
\text { application date }\end{array}$ & $\begin{array}{l}\text { Publication } \\
\text { date }\end{array}$ \\
\hline WO 2012096015 & $\begin{array}{l}\text { Nucleic acid information processing device and processing } \\
\text { method thereof. }\end{array}$ & $\begin{array}{l}\text { Japan Software } \\
\text { Management Co. Ltd. } \\
\text { (Japan), Bioinformatics } \\
\text { Institute for Global Good } \\
\text { (Tokyo), } \\
\text { Nasu H, } \\
\text { Tsujimoto A, Yamakawa } \\
\text { T, Ono H }\end{array}$ & $\begin{array}{l}\text { Nasu H, } \\
\text { Tsujimoto A, } \\
\text { Yamakawa T, } \\
\text { Ono H }\end{array}$ & $1 / 11 / 2011$ & $7 / 19 / 2012$ \\
\hline WO 2011153372 & $\begin{array}{l}\text { Methods and systems for simulations of complex biological net- } \\
\text { works using gene expression indexing in computational models. } \\
\text { A method has been developed for using genome-wide transcrip- } \\
\text { tion profile (that is, gene-expression level) values to derive a } \\
\text { gene expression index used as a kinetic value for every biological } \\
\text { reaction and process assigned to each and every gene. }\end{array}$ & $\begin{array}{l}\text { University of } \\
\text { Texas (Austin, TX, USA), } \\
\text { Phelix CF }\end{array}$ & Phelix CF & $6 / 2 / 2010$ & $12 / 8 / 2011$ \\
\hline US 2011172981 & $\begin{array}{l}\text { High-throughput, ensemble-based docking and elucidation of } \\
\text { three-dimensional structural confirmations of flexible } \\
\text { biomolecular targets. }\end{array}$ & $\begin{array}{l}\text { University of } \\
\text { Michigan } \\
\text { (Ann Arbor, MI, USA) }\end{array}$ & $\begin{array}{l}\text { Al-Hashimi } \\
\text { Hashim M, } \\
\text { Stelzer A, } \\
\text { Andricioaei I, } \\
\text { Aaron F }\end{array}$ & $9 / 26 / 2008$ & $7 / 14 / 2011$ \\
\hline WO 2011061548 & $\begin{array}{l}\text { A method for computational drug design using an evolutionary } \\
\text { algorithm, comprising evaluating virtual molecules according to } \\
\text { vector distance (VD) to at least one achievement objective that } \\
\text { defines a desired ideal molecule. }\end{array}$ & $\begin{array}{l}\text { University of Dundee } \\
\text { (UK), Besnard J }\end{array}$ & Besnard J & $11 / 20 / 2009$ & $5 / 26 / 2011$ \\
\hline CA 2769045 & $\begin{array}{l}\text { Method for binding-site identification by ligand competitive } \\
\text { saturation simulation. }\end{array}$ & $\begin{array}{l}\text { University of } \\
\text { Maryland } \\
\text { (College Park, MD, USA) }\end{array}$ & $\begin{array}{l}\text { Mackerell AD, } \\
\text { Guvench } 0\end{array}$ & $5 / 4 / 2009$ & $11 / 11 / 2010$ \\
\hline WO 2009120371 & Computationally designed inhibitors of amyloidosis. & $\begin{array}{l}\text { University of Washington } \\
\text { (Seattle), Daggett V, } \\
\text { Law P }\end{array}$ & Daggett V, Law P & $3 / 27 / 2008$ & $10 / 1 / 2009$ \\
\hline
\end{tabular}

Source: US Patent and Trademark Office, Espacenet, JP and EP patent offices. The status of each application is slightly different from country to country. 